Boise State University

ScholarWorks

Biomolecular Research Center Publications and

Presentations

Biomolecular Research Center

$10-1-2010$

\title{
The Expression Patterns of Minor Fibrillar Collagens During Development in Zebrafish
}

Ming Fang

Boise State University

Jason S. Adams

Boise State University

B. Lane Memahhan

Boise State University

Raquel J. Brown

Boise State University

Julia Oxford

Boise State University

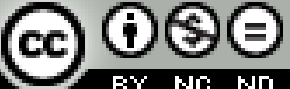

This is an author-produced, peer-reviewed version of this article. (C) 2009, Elsevier. Licensed under the Creative Commons AttributionNonCommercial-NoDerivatives 4.0 International License (https://creativecommons.org/licenses/by-nc-nd/4.0/). The final, definitive version of this document can be found online at Gene Expression Patterns, doi: 10.1016/j.gep.2010.07.002 


\title{
The Expression Patterns of Minor Fibrillar Collagens During Development in Zebrafish
}

\author{
Ming Fang, Jason S. Adams, B. Lane Mcmahan, Raquel J. Brown, and Julia Thom Oxford \\ Boise State University
}

\begin{abstract}
Minor fibrillar collagens are recognized as the organizers and nucleators during collagen fibrillogenesis but likely serve additional functions. The minor fibrillar collagens include collagens type V and type XI. Mutations of collagen type V and XI can cause Ehlers Danlos, Stickler's, and Marshall's syndromes in human. We have characterized the spatiotemporal expression patterns of Col11a1, Col11a2, Col5a1 as well as Col5a 3 in zebrafish embryos by in situ hybridization. Col5a1 is expressed in developing somites, neural crest, the head mesenchyme, developing cranial cartilage, pharyngeal arches and vertebrae. Col5a3 is detected in the notochord, mesenchyme cells in the eyes and lens. Both Col11a1 and Col11a2 have similar expression patterns, including notochord, otic vesicle, and developing cranial cartilages. Zebrafish may therefore serve as a valuable vertebrate model system for the study of diseases associated with collagens type V and XI mutations.
\end{abstract}

Keywords: minor fibrillar collagens, zebrafish, development, in situ hybridization, Danio rerio

\section{Results and discussion}

Collagens are the predominant proteins in the extracellular matrix of animals. They play diverse roles in cell adhesion, differentiation, cell migration as well as tissue regeneration. Currently, there are at least 29 different collagens known (Söderhäll et al, 2007; Kadler et al, 2007). Generally, collagen fibrils are composed of three peptide alpha chains forming either heterotrimers or homotrimers. Depending on their functions and domain homology, collagens can be grouped into seven different types (Kadler et al, 2007). Collagen type I, II, III, V and XI as well as recently discovered XXIV and XXVII are fibril-forming collagens. Historically, the fibrillar collagens are further divided into major (I, II and III) and minor (V, XI, XXIV and XXVII) fibrillar collagens based on their relative abundance in the collagen fibrils. For collagen type $\mathrm{V}$, three different alpha chains have been characterized in human: COL5A1, COL5A2 and COL5A3. Likewise, collagen type XI also has three alpha chains, COL11A1, COL11A2 and COL11A3. However, COL11A3 is actually COL2A1 (alpha1 chain, type II), one of the major fibrilforming collagens. Additionally, another alpha chain has been identified in rat, Col5a4, is expressed in Schwann cells and shows a high similarity to Col5a3 (Chernousov et al, 2000). However, Col5a4 has not been reported for other species.

Minor fibril-forming collagens are composed of several domains, including the C-propeptide ( $\mathrm{NC1}$ or $\mathrm{CPP}$ ), a major helix (COL1) and minor helix (COL2) separated by a short sequence (NC2), and the N-terminal end (NC3). The $\mathrm{NC} 1$ or CPP domain is well conserved among fibrilforming collagens, whereas NC3 domain varies in terms of size, primary structure and subdomains (Ricard-Blum, 2005). In the minor fibrillar collagens, NC3 contains either a cysteinerich repeat domain (CRR) feature with 10 cysteines in the sequence (Col5a2), or a thrombospondin Nterminal-like (TSPN) domain adjacent to a variable region (VR) (Col5a1, Col5a3, Col11a1 and Col11a2). The structural difference between Col5a2 and the other minor fibrillar collagens is in agreement with recent phylogenetic analysis (Zhang et al, 2007). The VR, historically, is defined as the region of low homology among different collagen alpha chains. For both collagens type V and XI, the amino acid sequence of the VRs are rich in charged residues under physiological conditions. The VR in Col11a1 contains a heparan sulfate binding site (Warner et al, 2006). In human, mouse, rat and other species, Col11a1 and Col11a2 also exhibit extensive alternative splicing (AS) in the VR ( $\mathrm{Li}$ et al, 1995; Lui et al, 1996; Oxford et al, 1995; Tsumaki \& Kimura, 1995). Although there can be many different combinations of exons in the VR, the most abundant forms of Col11a1 detected are the spliceforms 
that include exons 6A-7-8, exons 6B-7, and exon 7 alone between the constitutively expressed exons 5 and 9 (Morris et al, 2000; Oxford et al, 1995); in the case of Col11a2, exon 6, exon 7 and exon 8 can be either included or excluded independently (Lui et al, 1996; Tsumaki and Kimura, 1995).

The importance of collagens has been showed by their profound influence in connective tissue formation and function. In human, mutations in collagen type $\mathrm{V}$ genes cause Ehlers-Danlos syndrome, resulting in hypermobility of joints (Richards et al, 1998; Schwarze et al, 2000); whereas mutations in collagen type XI can cause Marshall's or Stickler syndrome, both resulting in altered facial appearance, eye abnormalities, joint problems and hearing loss (Annunen et al, 1999; Chen et al, 2005; McGuirt et al, 1999; Melkoniemi et al, 2000; Pihlajamaa et al, 1998; SirkoOsadsa et al, 1998). However, the pathogenic mechanisms for these syndromes are still not completely understood.

To date, there is little information about the early expression patterns of minor fibrillar collagens during early embryogenesis in vertebrate model organisms. Minor fibrillar collagens are expressed in a variety of different tissues in mouse, chicken, and rat. Col5a1 expression is observed in the aorta, heart, branchial arches, developing mesenchyme and neuroepithelium; later in development, Col5a1 expression is limited to bones, vertebral column and cornea as well as the tendons and ligaments (Imamura et al, 2000; Roulet et al, 2007). The location of expression of Col5a3 has been shown as a subset of that of Col5a1 (Imamura et al, 2000). Col11a1 has been detected in limbs, vertebrae, mandibular bones, otic vesicle, and the atrioventricular valve in the heart, neuroepithelium of the brain, liver, kidney, lung, muscle and intestine (Lui et al, 1995; Oxford et al, 1995; Yoshioka et al, 1995), mesothelial layer of the cornea, and perioptic mesenchyme within the sclera (Sugimoto et al, 1998). Relative to Col11a1, Col11a2 has more restricted cartilage-related expression in developing limbs and axial skeleton (Sugimoto et al, 1998). To further investigate the mechanisms of pathogenesis due to mutations in minor fibrillar collagens, we have characterized their spatial and temporal expression patterns during early development in zebrafish (Danio rerio). Recently, work by Baas and colleagues demonstrated the importance of one of the minor fibrillar collagens located on chr19 during embryonic development (Baas et al. 2009). Col11a2 expression pattern in embryonic structures was also described by Yokoi and colleagues (Yokoi et al. 2009). Results from our study are compared to the results of a recent studied carried out by Hoffman and colleagues (Hoffman et al. 2010) in which they characterized early expression patterns of type V and XI collagens.

\subsection{Characterization of zebrafish minor fibrillar collagens and proteins}

To identify zebrafish minor fibrillar collagens (Col5a1, Col5a3, Col11a1 and Col11a2), the NC1 or CPP domain of human minor fibrillar collagens were used to search against the GenBank and Ensembl databases using the Blastp search algorithm (WU Blast2.0 default settings). Loci identified as encoding both the NC1 domain and the amino terminal thrombospondin-like domain were further analyzed for predicted exons. Predicted exons were identified in the database and compared to other vertebrate species. To confirm predicted exons, PCR primers were designed, synthesized, and used to amplify the predicted variable regions by RT-PCR. Identity and exon composition were verified by sequencing DNA contained within excised bands. Accession numbers and the primer information are listed in Table 1. Our analysis focused on the alternative splicing in the VR.

Translation of the VRs of the minor fibrillar collagens were then compared among zebrafish, chimpanzee, mouse, rat and chicken. The level of conservation at the amino acid level is highlighted in Fig. 1, focusing on each exon in the VR. Although the VR demonstrates low homology among species, there are specific conserved patterns of residues that have been identified. Exon 6 (E6) in Col5a1 and E6A, E8 in Col11a1 as well as E8 in Col11a2 are rich in tyrosine and acidic residues aspartic acid and glutamic acid; whereas E6 in Col5a3 and E6B in Col11a1 include clusters of arginines and lysines. Tyrosine of Col5a1 is known to be extensively sulfonated in the extracellular matrix, and successive arginines and lysines have been shown to serve as a binding motif for proteoglycans (Erdman et al, 2002; Yamaguchi et al, 2005).

However, none of the tyrosine-rich or arginine/lysine-rich domains are thoroughly understood with respect to specific function in the minor collagens. A gene encoding a minor fibrillar collagen most closely related to Col11a1 (designated Col11alb by Hoffman et al., 2010) was identified on chromosome 2. No indication of alternative splicing was observed for the VR of Col11a1b in our study. 


\subsection{Expression of Col5a1, Col5a3, Col11a1 and Col11a2 during zebrafish development Temporal expression patterns.}

We used RT-PCR to determine the temporal expression patterns of Col5a1, Col5a3, Col11a1 and Col11a2. The transcription of all minor fibrillar collagen genes was first apparent at approximately $4-6 \mathrm{hpf}$, with the exception of Col11a2, in which maternal transcripts were detected (Supplement Fig. 1). Both Col11a1 and Col11a2 exhibited similar trends, demonstrating additional spliceforms at later developmental stages (Fig. 2). Sequence analysis of each band generated by PCR confirmed alternative splicing for these transcripts, which are likely to be developmentally regulated. In addition, we observed a previously undescribed exon within VR of Col11a2 in addition to exon 6,7 or 8 , which was between exon 6 and exon 7 in the genomic sequence. This exon (designated exon 6') was approximately 1200bp in length. Surprisingly, our experiments also demonstrated alternative splicing for Col5a3 on chr3 within the VR (Fig. 2A), which contained an additional 447bp exon. Alternative splicing within the VR of Col5a3 has not been reported for other species. This observation is in agreement with Hoffman and colleagues (Hoffman et al, 2010). The alternative splicing is summarized in Fig. 2B. Sequence data for each of the exons within the VRs is shown in Fig. 2C.

Spatial expression patterns. The spatial expression patterns of the minor fibrillar collagens were then examined by in situ hybridization. Antisense riboprobes were synthesized based on the mRNA sequences in the VR for each gene. Because Col5a1 does not show alternative splicing within the VR, the antisense riboprobe was designed to be complimentary to exons 6-7-8. However, as Col5a3, Col11a1a and Col11a2 undergo alternative splicing within the VR, the riboprobes were designed from the earliest or most prevalent spliceform. For Coll1a1a, the probe was designed to hybridize to exons 6a-7-8, which is the major product demonstrated by RT-PCR (Fig. 2). Detection of Col5a3 and Col11a1b was accomplished using probes representing all exons within the variable region.

Generally, Col5a1 was expressed in developing somites, neural crest, and head mesenchyme at early timepoints; at later stages, it was expressed in the developing cranial cartilage, pharyngeal arches and vertebrae. Col5a 3 was detected in the notochord at early timepoints in development, and later in mesenchymal cells of the eyes and lens. Col11a1a and Col11a2 demonstrated similar expression patterns, including notochord, otic vesicle, and developing cranial cartilages. Interestingly, Col11a1b on chromosome 2 shared a very similar expression pattern with Col5a1.

Comparative expression at $16 \mathrm{hpf}$. At $16 \mathrm{hpf}$, Col5a1 was expressed predominantly in the cranial neural crest and in the region of developing somites (Fig. 3A, F, K). Col5a3 was expressed in the notochord (Fig. 3B, G), as was Col11a1a on chromosome 24 (Fig. D, I) and Col11a2 (Fig. 3E, J). Col11a1b on chromosome 2 was detected near the otic placode and hindbrain (Fig. 3C, H, L) as was Col11a1a on chromosome 24 (Fig.3D, I, M). Col11a2 was expressed around the developing notochord (Fig. 3E, J). Controls showed no detectable signal.

Comparative expression at 24 hpf. At $24 \mathrm{hpf}$, Col5a1 was detected in the locations observed at $16 \mathrm{hpf}$ (Fig. 4A, B, C), and also in agreement with expression patterns observed in mouse (Roulet et al, 2007). Col5a3 was expressed in the notochord with a stronger signal toward the tip of the tail. Col11a1b on chromosome 2 seemed to have a similar pattern as Col5a1 at this time point although weaker. Both Col11a1a on chromosome 24 and Col11a2 were detected in the notochord. The previously observed expression of Col11a1a on chromosome 24 in otic placode and hindbrain was not detected at $24 \mathrm{hpf}$, indicating transient expression in the hindbrain and otic placode during early development. Marshall's and Stickler syndrome patients commonly have hearing problems, and this transient expression may shed light on the mechanism of how mutations in Col11a1 are associated with these symptoms. Col11a2 was detected faintly in the region of the developing eye (Fig. 4C right-hand column). Controls showed no detectable signal.

Comparative expression at 48 and 72 hpf. At 48 and 72 hpf, the expression of Col5a1 was detected in the region of developing somites, perioptic mesenchyme cells in the eyes, hindbrain and otic vesicle (Fig. 5A, B). Col5a3 was expressed at $48 \mathrm{hpf}$ at the posterior end of the notochord, the lens and mesenchyme cells of the eyes (Fig. 5A, B). In addition, Col5a3 also was expressed in the developing anterior pericardium (Fig. 5B, second column). Col11a1b on chromosome 2 showed a similar expression pattern to Col5a1 in the region of the developing somites (Fig. 5B first and third columns). Expression of both Col11a1a on chromosome 24 and Col11a2 was detected in the notochord, in the region of the otic vesicles, and in the developing ethmoid plate and trabeculae. (Fig. 5 and 6). Col11a1a was expressed in polar cartilage, whereas Col11a2 exhibited strong expression within the parachordal cartilage. 
At $72 \mathrm{hpf}$, Col5a1 had a similar expression patterns as that observed for $48 \mathrm{hpf}$ (Fig. 6AE). Additionally, Col5a1 was detected strongly in the parachordal cartilage, ceratohyal, trabeculae, and madibular cartilage, the outlining of the cranial cavity (Fig. 6A, D). The expression of Col5a3 was detectable in the notochord, in the region of the retinal pigmented epithelium, and lens (Fig. 6A, B, D). Col11a1b on chromosome 2 demonstrated similar expression patterns as those of Col5a1 (Fig. 6, column 1 and 3). Both Col11a1a on chromosome 24 and Coll1a2 were expressed in the notochord, ceratohyal, palatoquadrate and Meckel's cartilage in addition to those structures that were detected at $48 \mathrm{hpf}$ (Fig. 6 column 4 and 5). Controls showed no significant signal at all time points.

\subsection{Summary}

Our study has identified orthologs of minor fibrillar collagens in zebrafish, characterizing and contrasting the expression patterns. Col11a1a on chromosome 24 and Col11a1b on chromosome 2 as well as Col5a1 have been extensively characterized during early development in zebrafish by our study. For Col5a1, the strong expression in the neural crest, head mesenchyme and in the region of developing somites may indicate a broad spectrum of functions. Sequence alignment and synteny analysis suggest that the gene on chromosome 2 is most closely related to Col11a1. Our results show that this gene does not undergo alternative splicing within the VR, and exhibits a similar expression pattern to that of Col5a1. Interestingly, we have identified transient expression of Col11a1a on chromosome 24 around $16 \mathrm{hpf}$, which includes hindbrain and otic placode. This finding has not been reported in other species. With regard to the collagenopathies of Col11a1 in humans, the transient expression in hindbrain and otic placode may contribute to the clinical symptoms that have been described in Marshall's and Stickler syndromes. The developmental events associated with transient expression require further investigation.

Baas and colleagues reported the identification and characterization of collagen XI cDNA using zebrafish genomic sequence reported to be the ortholog of human COL11A2 (GenBank accession number AL672176 and XM685088). Within the coding sequence, they identified characteristic features of all minor fibrillar collagens, a 60 amino acid variable region, and a carboxyl terminal domain with the length, number and location of cysteine residues within the $\mathrm{NC} 1$ domain that is more consistent with the identity as an ortholog of human COL11A1. (Baas et al 2009.) Overall amino acid sequence identity however, indicates that this minor fibrillar collagen alpha chain is more likely to be an ortholog of human COL11A2. Sequence comparison of the six amino acids between cysteines 3 and 4 of the amino propeptide domain was also cited as supporting the identification of this minor fibrillar collagen gene as Col11a1 rather than the previous identification as Col11a2. Both COL11A1 and COL11A2 play critical roles in vertebrate craniofacial development and mutations in either of these genes can result in Stickler syndrome. Therefore, it is possible that the in situ hybridization data and characterization of the morphants carried out by Baas and colleagues may actually reflect the essential role that Col11a2 plays in craniofacial development and morphogenesis in the zebrafish rather than Col11a1, as their title indicates. It is possible that all minor fibrillar collagens belonging to the V/XI collagen family share common function as well as serving unique roles in tissue and species-specific manners.

Additional findings from our study include the detection of exon $6 \mathrm{~b}$ of Col11a1a at $72 \mathrm{hpf}$. While not reported in previously related work (Baas et al., 2009, Hoffman et al., 2010), sequence corresponding to exon $6 \mathrm{~b}$ was previously submitted to GenBank as a result of expression profiling and comparative genomics analysis (Dickmeis et al., 2004). Characterization of Col11a1b on chr2 in our study agrees with findings by Hoffman and colleagues (Hoffman et al., 2010). A short form of Col11a2 was reported by Hoffman and colleagues; here we report several spliceforms, including a form with an extensive alternatively spliced exon of approximately $1.2 \mathrm{~kb}$ (termed exon 6 ' in our current study). While expression of this exon has not been previously reported, sequence corresponding to this region was included in the entry from Howden (Howden, P. Direct Submission, Submitted (12-JAN-2009) Wellcome Trust Sanger Institute, Hinxton, Cambridgeshire, CB10 1SA, UK). Results from our laboratory agree with those reported by Hoffman and colleagues for Col5a1, and to a large extent for Col5a3 with only minor sequence differences.

\section{Experimental procedures}

\subsection{Zebrafish care}

Zebrafish were maintained and bred under conditions described by Westerfield \{Westerfield, 1995\}. Embryos were collected and staged according to hour post-fertilization (hpf). Prior to in situ hybridization, fixed embryos were treated with $3 \% \mathrm{H} 2 \mathrm{O} 2$ in methanol for $0.5-1$ hour to remove the pigment. 


\subsection{RT-PCR and cloning}

Col5a1 and Col11a2 are annotated in ENSEMBL website (www.ensembl.org), whereas Col5a3 and Col11a1 were not available at the time of these experiments. The candidates for zebrafish Col5a3 and Col11a1 orthologs were identified by Blastp search with human COL5A3 and COL11A1 translated sequences. Total RNA was extracted from $4 \mathrm{hpf}, 6 \mathrm{hpf}$, 10hpf, 16hpf, 24hpf, 36hpf, 48hpf zebrafish embryos by using TRIZOL reagent (Invitrogen, US) and reverse transcribed with random decamers and MMLV retro-transcriptase (Invitrogen, US) according to manufacturer's protocols. The sequences extending from exon 5 to exon 9 of each gene was amplified by using PuReTaq Ready-To-Go PCR beads (GE Healthcare, US) and cloned into pCRII vector (Invitrogen, US) for sequencing and riboprobe synthesis. Primer sequences and the annealing temperatures are listed in Table 1. DNA sequencing of excised bands and cloned fragments was carried out at Idaho State University, Molecular Research Core Facility.

\subsection{Whole mount in situ hybridization, sectioning and imaging}

Antisense digoxigenin labeled riboprobes for Col5a1, Col5a3, Col11a1a, Col11a1b and Col11a2 were synthesized by DIG RNA labeling kit (Roche, US). Standard procedure followed manufacturer's instruction. Probes were further purified by using Micro Bio-spin columns (Bio-Rad, US). A negative control probe was synthesized from liberalized pSPT-18-neo plasmid by using the same procedures. Whole mount in situ hybridization was performed as previously described (Bisgrove et al, 1999). Images were captured with a Stemi SV11 system (Zeiss, US). For sectioning, we followed procedures that have been previously described by (Zhou and Hildebrandt, 2009). Ten micrometer sections were obtained using Sorvall JB-4 microtome. Images were taken with Zeiss Stemi SV11 Apo microscope and Axio-CAM digital camera.

\section{Acknowledgements}

Authors acknowledge the technical support of Linda Mercer and Dawn Mikelonis. This work was supported in part by grants from the Arthritis Foundation, NIH (RO1AR47985, R15HD059949 and KO2AR48672), by a grant from NIH/NCRR (P20RR16454), the National Science Foundation (0321233), Idaho State Board of Education funding for the Musculoskeletal Research Institute, NASA Idaho Space Grant Consortium (JSA), University of Washington WWAMI MSRTP (BLM) Department of Biological Sciences Graduate Student Fellowship (MF), funding from the M.J. Murdock Foundation and from Lori and Duane Stueckle. 


\section{References}

Annunen S, Körkkö J, Czarny M, Warman ML, Brunner HG, Kääriäinen H, Mulliken JB, Tranebjæg L, Brooks DG, Cox GF, Cruysberg JR, Curtis MA, Davenport SLH, Friedrich CA, Kaitila I, Krawczynski MR, Latos-Bielenska A, Mukai S, Olsen BR, Shinno N, Somer M, Vikkula M, Zlotogora J, Prockop DJ, Ala-Kokko L (1999) Splicing mutations of 54-bp exons in the COL11A1 gene cause Marshall syndrome, but other mutations cause overlapping Marshall/Stickler phenotypes. Am J Hum Genet 65: 974-983

Baas D, Malbouyres M, Haftek-Terreau Z, Le Guellec D, Ruggiero F. (2009) Craniofacial cartilage morphogenesis requires zebrafish col11a1 activity. Matrix Biol. 28:490-502

Bisgrove BW, Essner JJ, Yost HJ (1999) Regulation of midline development by antagonism of lefty and nodal signaling. Development 126: 3253-3262

Chen W, Kahrizi K, Meyer NC, Riazalhosseini Y, Van Camp G, Najmabadi H, Smith RJH (2005) Mutation of COL11A2 causes autosomal recessive non-syndromic hearing loss at the DFNB53 locus. J Med Genet 42: e61

Chernousov MA, Rothblum K, Tyler WA, Stahl RC, Carey DJ (2000) Schwann Cells Synthesize Type V Collagen That Contains a Novel $\alpha 4$ Chain. Journal of Biological Chemistry 275: 28208- 28215

Dickmeis T, Plessy C, Rastegar S, Aanstad P, Herwig R, Chalmel F, Fischer N, Strahle U (2004) Expression profiling and comparative genomics identify a conserved regulatory region controlling midline expression in the zebrafish embryo. Genome Res. 14: 228-238

Erdman R, Stahl RC, Rothblum K, Chernousov MA, Carey DJ (2002) Schwann cell adhesion to a novel heparan sulfate binding site in the N-terminal domain of alpha 4 type $\mathrm{V}$ collagen is mediated by syndecan-3. J Biol Chem 277: $7619-7625$

Hoffman GG, Branam AM, Huang G, Pelegri F, Cole WG, Wenstrup RM, Greenspan DS Characterization of the six zebrafish clade B fibrillar procollagen genes, with evidence for evolutionarily conserved alternative splicing within the pro-[alpha]1(V) C-propeptide. Matrix Biology In Press.

Imamura Y, Scott IC, Greenspan DS (2000) The pro-alpha 3(V) Collagen Chain. Complete primary structure, expression domains in adult and developing tissues, and comparison to the structures and expression domains of the other types V and XI procollagen chains. J Biol Chem 275: 8749-8759

Kadler KE, Baldock C, Bella J, Boot-Handford RP (2007) Collagens at a glance. J Cell Sci 120: 1955-1958

Li Y, Lacerda DA, Warman ML, Beier DR, Yoshioka H, Ninomiya Y, Oxford JT, Morris NP, Andrikopoulos K, Ramirez F, Wardell BB, Lifferth GD, Teuscher C, Woodward SR, Taylor BA, Seegmiller RE, Olsen BR (1995) A fibrillar collagen gene, Col11a1, is essential for skeletal morphogenesis. Cell 80: 423-430

Lui VC, Kong RY, Nicholls J, Cheung AN, Cheah KS (1995) The mRNAs for the three chains of human collagen type XI are widely distributed but not necessarily co-expressed: implications for homotrimeric, heterotrimeric and heterotypic collagen molecules. Biochem J 311: 511-516

Lui VH, Ng L, Sat EY, Nicholls J, Cheah KE (1996) Extensive alternative splicing within the amino-propeptide coding domain of alpha $2(\mathrm{XI})$ procollagen mRNAs. Expression of transcripts encoding truncated pro-alpha chains. $J$ Biol Chem 271: 16945-16951

McGuirt WT, Prasad SD, Griffith AJ, Kunst HPM, Green GE, Shpargel KB, Runge C, Huybrechts C, Mueller RF, Lynch E, King M-C, Brunner HG, Cremers CWRJ, Takanosu M, LiS-W, Arita M, Mayne R, Prockop DJ, Camp GV, Smith RJH (1999) Mutations in COL11A2 cause non-syndromic hearing loss (DFNA13). Nat Genet 23: 413419 
Melkoniemi M, Brunner HG, Manouvrier S, Hennekam R, Superti-Furga A, Käriäinen H, Pauli RM, van Essen T, Warman ML, Bonaventure J, Miny P, Ala-Kokko L (2000) Autosomal recessive disorder otospondylomegaepiphyseal dysplasia is associated with loss-of-function mutations in the COL11A2 gene. Am J Hum Genet 66: 368-377

Morris NP, Oxford JT, Davies GBM, Smoody BF, Keene DR (2000) Developmentally regulated alternative splicing of the $\alpha 1$ (XI) collagen chain: spatial and temporal segregation of isoforms in the cartilage of fetal rat long bones. $J$ Histochem Cytochem 48: 725-742

Oxford JT, Doege KJ, Morris NP (1995) Alternative exon splicing within the amino-terminal nontriple-helical domain of the rat pro- $\alpha 1(\mathrm{XI})$ collagen chain generates multiple forms of the mRNA transcript which exhibit tissuedependent variation. J Biol Chem 270: 9478-9485

Pihlajamaa T, Prockop DJ, Faber J, Winterpacht A, Zabel B, Giedion A, Wiesbauer P, Spranger J, Ala-Kokko L (1998) Heterozygous glycine substitution in the COL11A2 gene in the original patient with the WeissenbacherZweymüler syndrome demonstrates its identity with heterozygous OSMED (nonocular Stickler syndrome). Am $J$ Med Genet 80: 115-120

Richards AJ, Martin S, Nicholls AC, Harrison JB, Pope FM, Burrows NP (1998) A single base mutation in COL5A2 causes Ehlers-Danlos syndrome type II. J Med Genet 35: 846-848

Roulet M, Ruggiero F, Karsenty G, LeGuellec D (2007) A comprehensive study of the spatial and temporal expression of the col5a1 gene in mouse embryos: a clue for understanding collagen $\mathrm{V}$ function in developing connective tissues. Cell Tissue Res 327: 323-332

Schwarze U, Atkinson M, Hoffman GG, Greenspan DS, Byers PH (2000) Null alleles of the COL5A1 gene of type V collagen are a cause of the classical forms of Ehlers-Danlos syndrome (types I and II). Am J Hum Genet 66: 17571765

Sirko-Osadsa DA, Melissa AM, Jennifer AS, Mary Ann L, Matthew LW, Nathaniel HR (1998) Stickler syndrome without eye involvement is caused by mutations in COL11A2, the gene encoding the $\alpha 2$ (XI) chain of type XI collagen. J Pediatr 132: 368-371

Söderhäll C, Marenholz I, Kerscher T, Schendorf F, Esparza-Gordillo J, Worm M, Gruber C, Mayr G, Albrecht M, Rohde K, Schulz H, Wahn U, Hubner N, Lee Y-A (2007) Variants in a novel epidermal collagen gene (COL29A1) are associated with atopic dermatitis. PLoS Biology 5: e242

Sugimoto M, Kimura T, Tsumaki N, Matsui Y, Nakata K, Kawahata H, Yasui N, Kitamura Y, Nomura S, Ochi T (1998) Differential in situ expression of alpha2(XI) collagen mRNA isoforms in the developing mouse. Cell Tissue Res 292: 325-332

Tsumaki N, Kimura T (1995) Differential expression of an acidic domain in the amino-terminal propeptide of mouse pro-alpha2(XI) collagen by complex alternative splicing. J Biol Chem 270: 2372-2378

Warner LR, Brown RJ, Yingst SMC, Oxford JT (2006) Isoform-specific heparan sulfate binding within the amino terminal noncollagenous domain of collagen alpha 1 (XI). J Biol Chem 281: 39507-16

Yamaguchi K, Matsuo N, Sumiyoshi H, Fujimoto N, Iyama K-I, Yanagisawa S, Yoshioka H (2005) Pro- $\alpha 3(V)$ collagen chain is expressed in bone and its basic N-terminal peptide adheres to osteosarcoma cells. Matrix Biol 24: 283-294

Yokoi H, Yan YL, Miller MR, BreMiller RA, Catchen JM, Johnson EA, Postlethwait JH.(2009) Expression profiling of zebrafish sox 9 mutants reveals that Sox9 is required for retinal differentiation. Dev Biol. 329:1-15

Yoshioka H, Iyama K-I, Inoguchi K, Khaleduzzaman M, Ninomiya Y, Ramirez F (1995) Developmental pattern of expression of the mouse alpha1(XI) collagen gene (Col11a1). Dev Dyn 204: 41-47 
Zhang X, Boot-Handford RP, Huxley-Jones J, Forse LN, Mould AP, Robertson DL, Li L, Athiyal M, Sarras MP, Jr. (2007) The Collagens of Hydra Provide Insight into the Evolution of Metazoan Extracellular Matrices. J Biol Chem 282: $6792-6802$

Zhou W, Hildebrandt F (2009) Molecular cloning and expression of phospholipase C epsilon 1 in zebrafish. Gene Expression Patterns 9: 282-288

\section{Figure Legends}

Figure 1. Sequence and domain structure alignment of minor fibrillar collagens in zebrafish (D. rerio), human $(H$. sapiens), mouse (M. musculus), rat ( $R$. norvegicus), chimpanzee ( $P$. troglodytes) and chicken ( $G$. gallus). Protein sequence alignment was generated by ClustalW algorithm, zebrafish sequences were predicted based on our sequencing results carried out in this study. A and C) Alignments of peptides encoded by exon 6,7 and 8 of Col5a1 and Col11a2, respectively; B) Alignment of peptides encoded by exon 6, 7 and 8 of Col5a3; Red arrows indicate the boundaries of exon 6-7 and exon 7-8, respectively; D) Alignments of peptides encoded by exon 6A, 6B, 7 and 8 of Col11a1. Exon assignments are made according to the human sequences, with the exception of Col5a1,exon 4, 5, 6, in which chicken exon sequences were used for the alignment of Col5a1_E6, Col5a1_E7 and Col5a1_E8, respectively; whereas for chimp, exon 7, 8 and 9 correspond to the exons of the VR. (A). Blue letters represent the conserved residue among species. Abbreviations: E6, exon6; E7, exon7; E8, exon8.

Figure 2. Temporal expression and splicing patterns of minor fibrillar collagens. RT-PCR was performed using RNA extracted from wild type embryos at 4, 6, 10, 16, 24, 36, 48, 72hpf. (A). Multiple bands were observed when cDNA was amplified within the region between exons 5 and 9 in the case of Col11a1a on chr24, Col11a2 on chr19, and Col5a3 on chr3. Single amplification products were observed in the case of Col5a1 and Col11a1b on chr2. (B). Splicing patterns are depicted, reflecting the exon usage for those genes undergoing alternative splicing within the variable region. Boxes represent individual exons within the VR, whereas lines in between represent introns. Relative size of all elements depicted reflects relative actual size in basepairs. For Col11a1a on chr24, the predominant spliceform at the earliest stages examined in this study included exons $6 \mathrm{~A}, 7$, and 8 within the variable region. Other minor transcripts observed included exons $6 \mathrm{~A}$ and $6 \mathrm{~B}$, or exon 7 alone. For Col11a2, exons 6,7 or 8 were observed to be either included or excluded in a complex pattern. An additional exon of approximately $1.2 \mathrm{~kb}$ was observed, replacing exons 6,7 and 8 in the case of the largest alternatively spliced form. In the case of Col5a3, alternative splicing involved exon 7. (C). Each amplification product was excised and sequenced to confirm identity, intron-exon boundaries, and exon usage in comparison to genomic sequence currently available. Variable region exon sequences in $\mathrm{C}$ are color-coded to match the block diagram in $\mathrm{B}$.

Figure 3. Spatial expression patterns of minor fibrillar collagen genes in zebrafish at 16 hpf by whole mount in situ hybridization. The expression patterns of Col5a1 (A, F, K), Col5a3 (B, G), Col11a1b (C, H, L), Col11a1a (D, I, M) and Col11a2 (E, J) are shown in both dorsal (A-E) and lateral (F-J) views. Arrows in D and H indicate expression in the hindbrain. Magnified views of A, H, and D demonstrate Col5a1 expression in neural crest (K), Col11a1b (L) and Col11a1a (M) in hindbrain (arrow in $\mathrm{L}$ and lower arrow in $\mathrm{M}$ ) and otic placode (upper arrow in $\mathrm{M}$ ).

Figure 4. Spatial expression patterns of minor fibrillar collagen genes in zebrafish at 24 hpf by whole mount in situ hybridization. The expression patterns of Col5a1, Col5a3, Col11a1b, Col11a1a, and Col11a2 are shown in both dorsal (A) and lateral (B) views. Magnified views demonstrate expression in notochord and region of developing somites (C). Magnified view in column 5, row $\mathrm{C}$ depicts Col11a2 expression in the developing eye.

Figure 5. Spatial expression patterns of minor fibrillar collagen genes in zebrafish at 48 hpf by whole mount in situ hybridization. The expression patterns of Col5a1, Col5a3, Col11a1b, Col11a1a, and Col11a2 are shown in both dorsal (A) and lateral (B) views. Expression is observed in the perioptic mesoderm, developing craniofacial cartilages, and presumptive retinal pigmented epithelium. Higher magnification views of patterns of expression are shown in Figure 6 for 72 hpf.

Figure 6. Spatial expression patterns of minor fibrillar collagen genes in zebrafish at $72 \mathrm{hpf}$ by whole mount in situ hybridization. The expression patterns of Col5a1, Col5a3, Col11a1b, Col11a1a, and Col11a2 are shown in dorsal (A), lateral (B and C), and ventral (D and E) views. A comparison of overall craniofacial staining is shown in A, C, and $\mathrm{D}$, demonstrating staining in the cranial cartilages including the trabeculae, ceratohyal, ethmoid plate, Meckel's 
NOTICE: This is the author's version of a work accepted for publication by Elsevier. Changes resulting from the publishing process, including peer review, editing, corrections, structural formatting and other quality control mechanisms, may not be reflected in this document. Changes may have been made to this work since it was submitted for publication. The definitive version has been published in Gene Expression Patterns, 2010. DOI: 10.1016/j.gep.2010.07.002

cartilage, polar cartilage, palatoquadrate, and parachordal cartilage. Expression in the eye is shown in row B. Anterior-most view of developing craniofacial region is shown in row E. Supplemental Figure 1. Expression of minor fibrillar collagens at $0 \mathrm{hpf}$ in zebrafish. Only Col11a2 shows maternal transcription.

Supplemental Figure 1. Expression of minor fibrillar collagens at 0 hpf in zebrafish. Only Col11a2 shows maternal transcription. 
Tables

Table 1. Accession numbers and primers for Col5a1, Col5a3, Col11a1, Col11a2.

\begin{tabular}{|c|c|c|c|}
\hline Gene & $\begin{array}{l}\text { GenBank } \\
\text { Accession \# }\end{array}$ & $\begin{array}{l}\text { Chromosome } \\
\text { location }\end{array}$ & Sequence \\
\hline Col5a1 & XM_685787.3 & 21 & $\begin{array}{l}\text { Forward: CTGATGATTGTATCGGACCAC } \\
\text { Reverse: GCTCGCCTTTCTGCCCTT }\end{array}$ \\
\hline Col5a3 & XM_001921860.1 & 3 & $\begin{array}{l}\text { Forward: TCTGACTACTGCCAAGATTAC } \\
\text { Reverse: TCTCCCTTCTGTCCTTTTG }\end{array}$ \\
\hline Coll1ala & NM_001083844.1 & 24 & $\begin{array}{l}\text { Forward: ATACAACAACTGCTGGATTGTGGC } \\
\text { Reverse: TGGTTCAATCACTGCAGGC }\end{array}$ \\
\hline Col11a1b* & XM_677653.3 & 2 & $\begin{array}{l}\text { Forward: GGGACATCCAGCAACTTCTTC } \\
\text { Reverse: CGGCTCAATGACTGCAGGTTC }\end{array}$ \\
\hline Col11a2 & NM_001079992.1 & 19 & $\begin{array}{l}\text { Forward: CAGCTGCTGATCGCATCCAAC } \\
\text { Reverse: GTCCTGGTGGTCCTTCAATCA }\end{array}$ \\
\hline
\end{tabular}

*Col11a1b is an additional possible ortholog of COL11A1 in zebrafish on chr2 
A

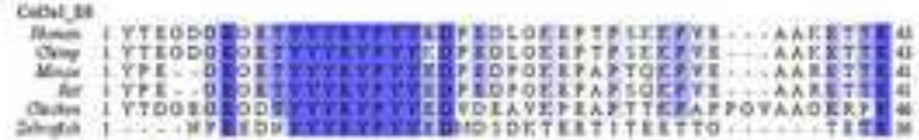

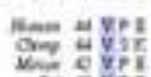

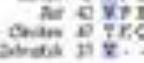

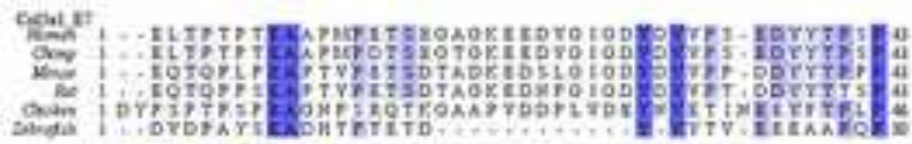

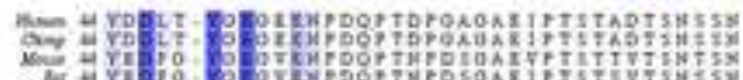

on dy

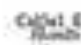

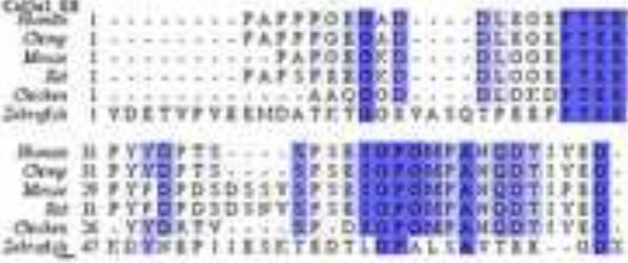

C

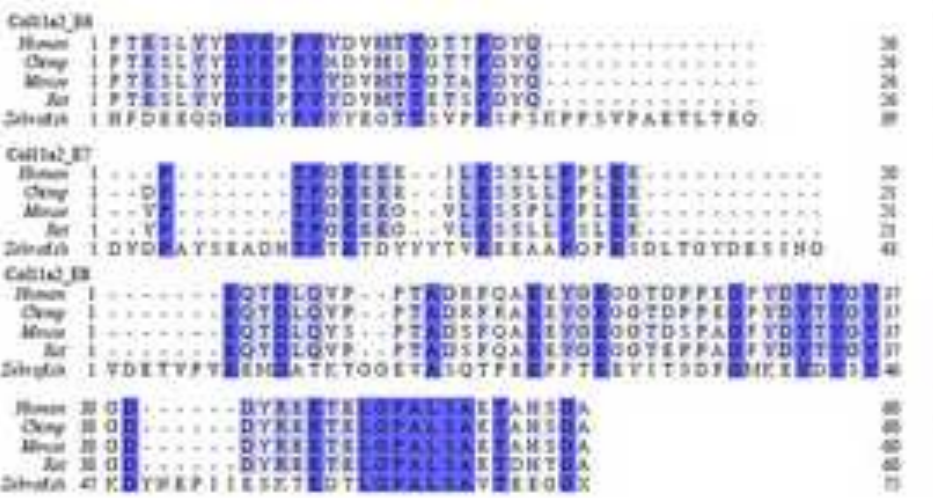

B

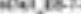

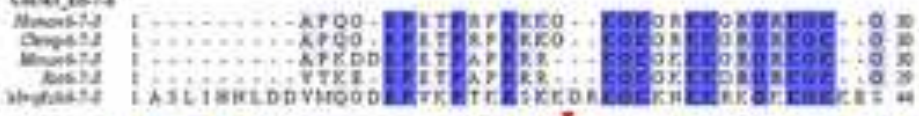

now i

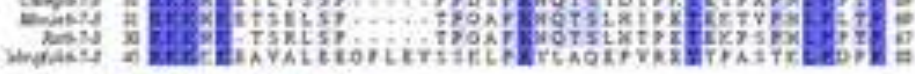

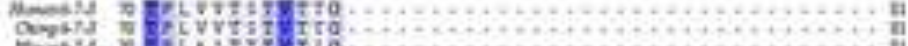

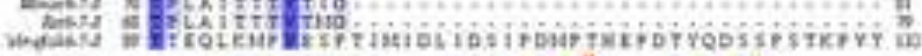

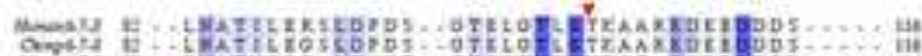

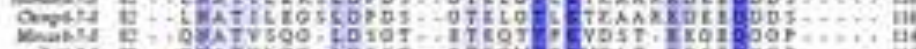
mosy

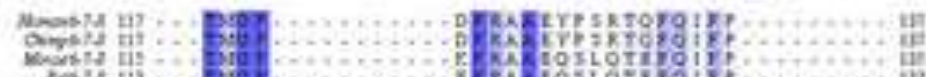
wdisu

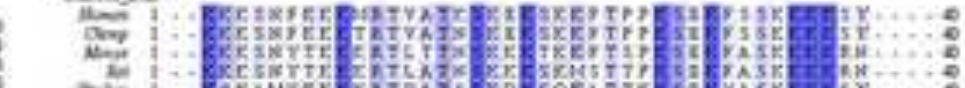
on in

는 f

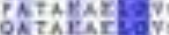

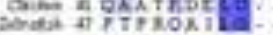

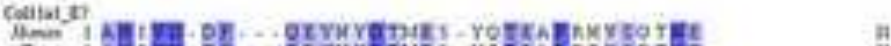
的

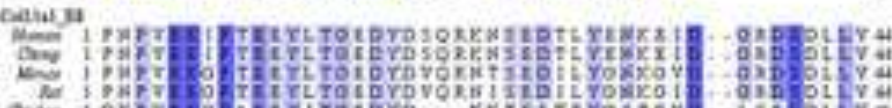
on

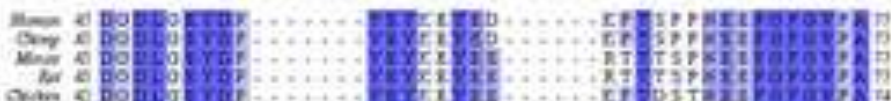

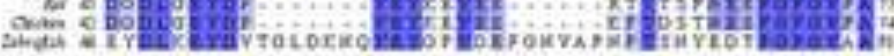

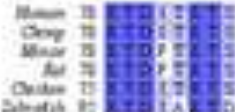


A

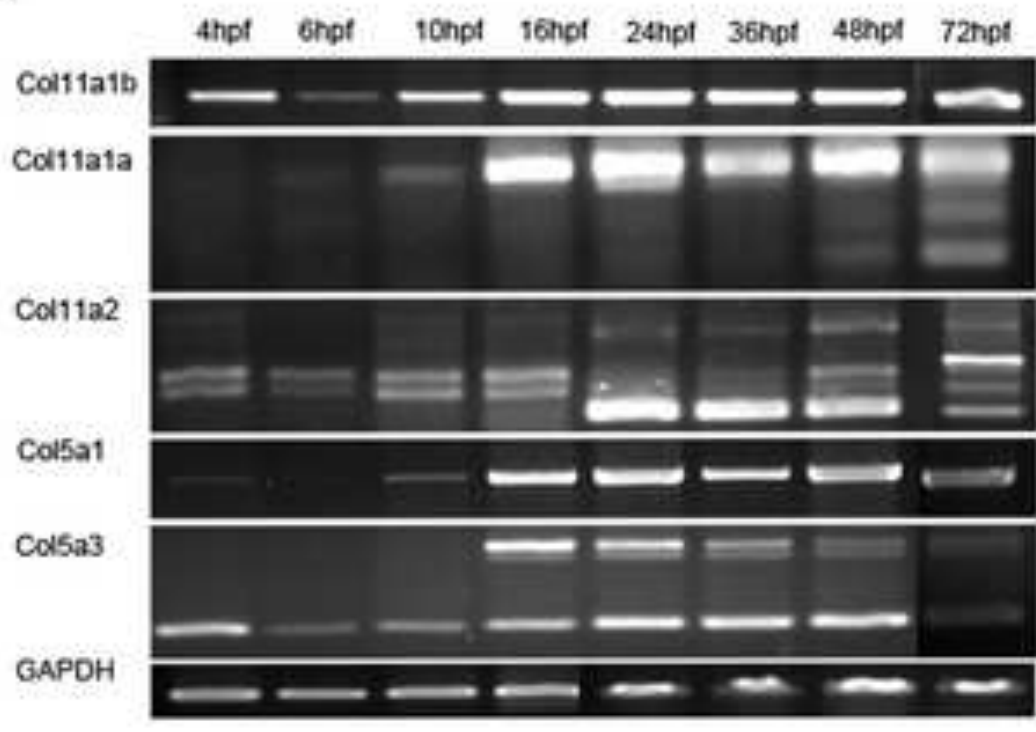

B

colstats

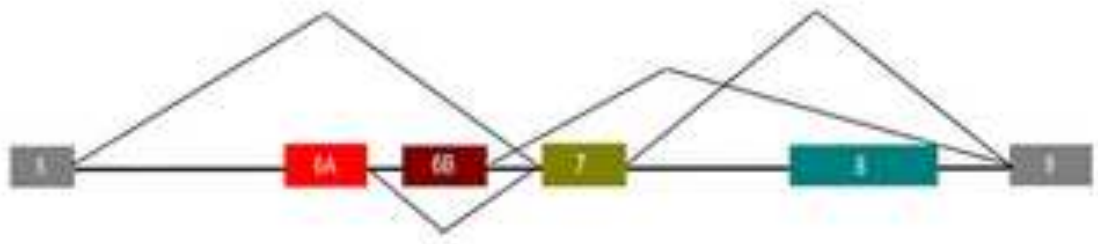

Cont22

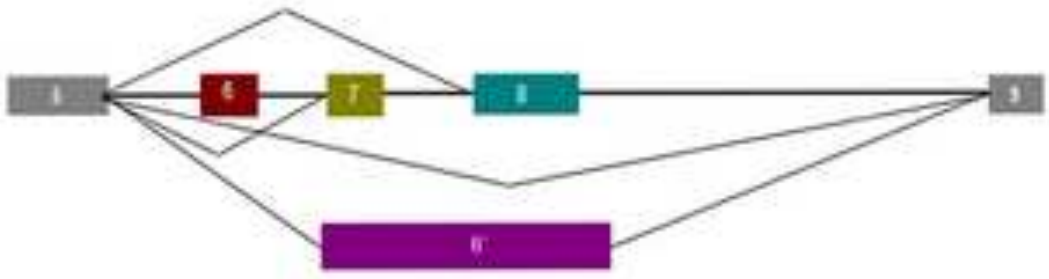

$\cos 43$

C

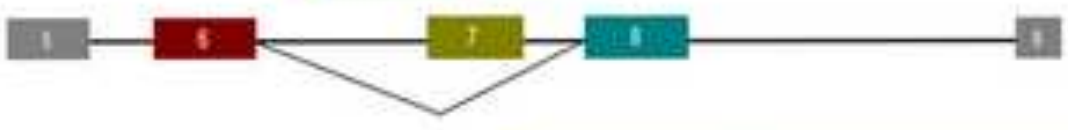

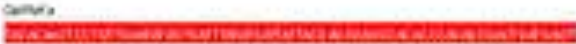

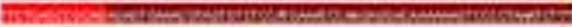

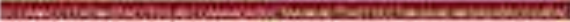

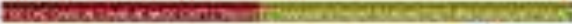

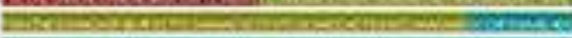

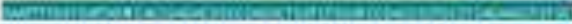

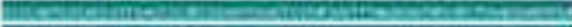

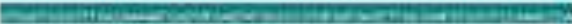

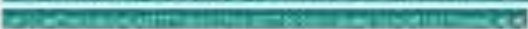
20

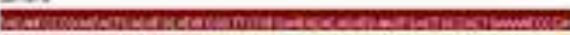

IA Fr

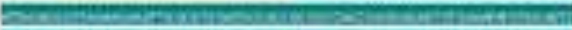

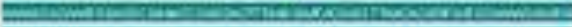
勇

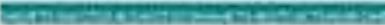

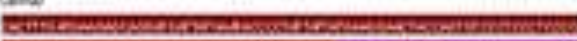

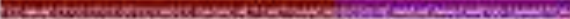

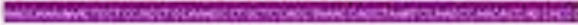
Fent

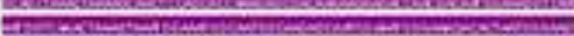

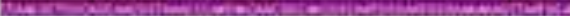

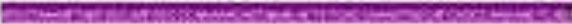
Cocrom Ecminum

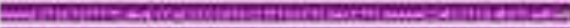

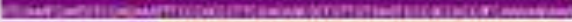

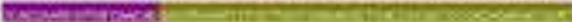

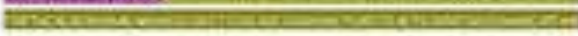

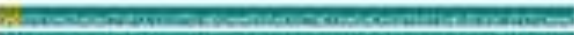

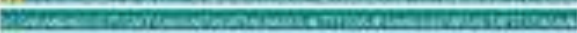

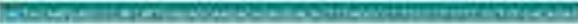
teicts

nat

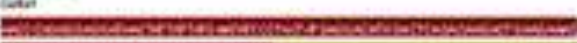

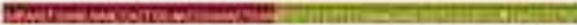

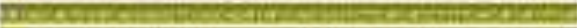

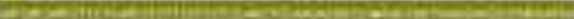

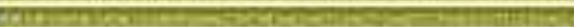

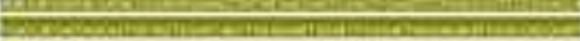

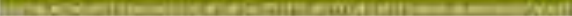

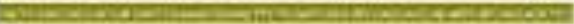

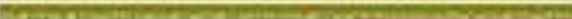

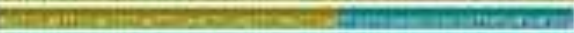

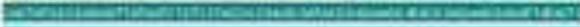

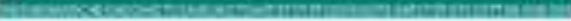
Fuscom Ple pow

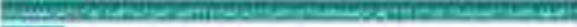
natser

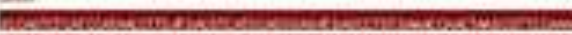

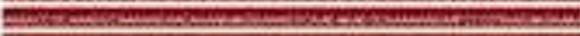

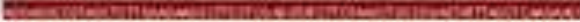
Phoweses:

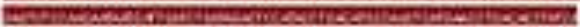

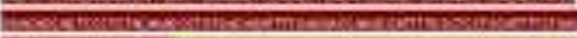

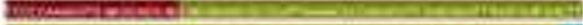

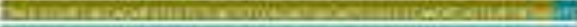

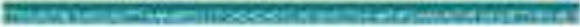

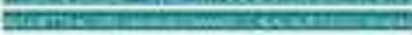




\section{dorsal side}

col5a1

A

B

col5a3

col11a1b
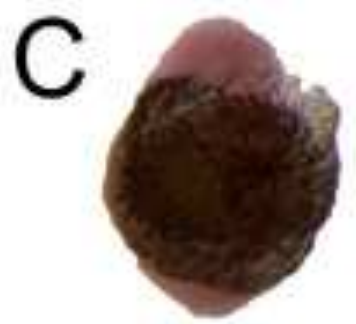

D

col11a1a

col11a2

E
$H$

$\mathrm{F}$

G
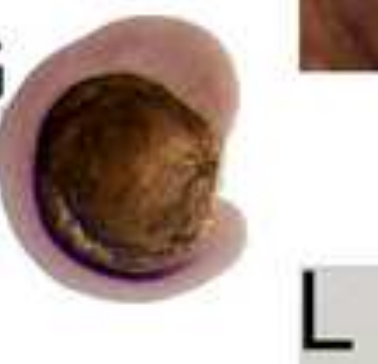

$\searrow$

M

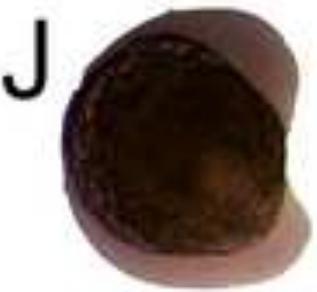

I

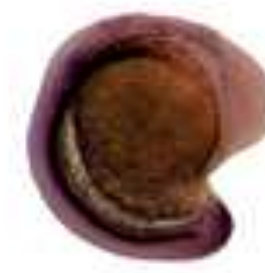

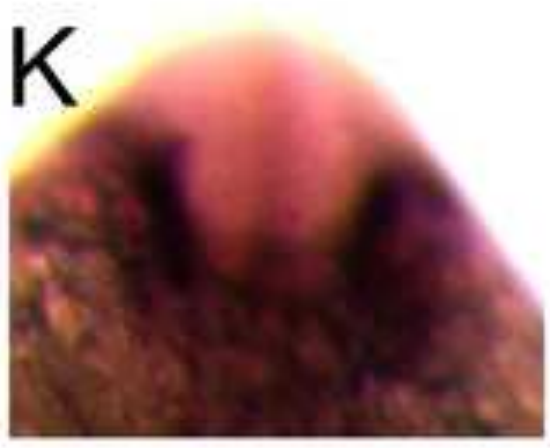
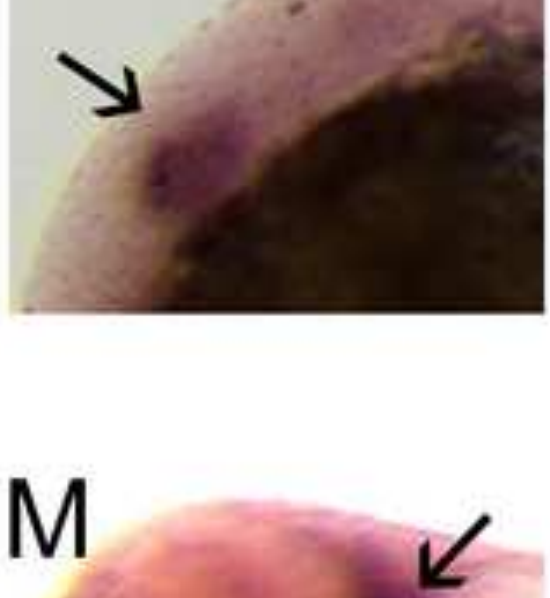
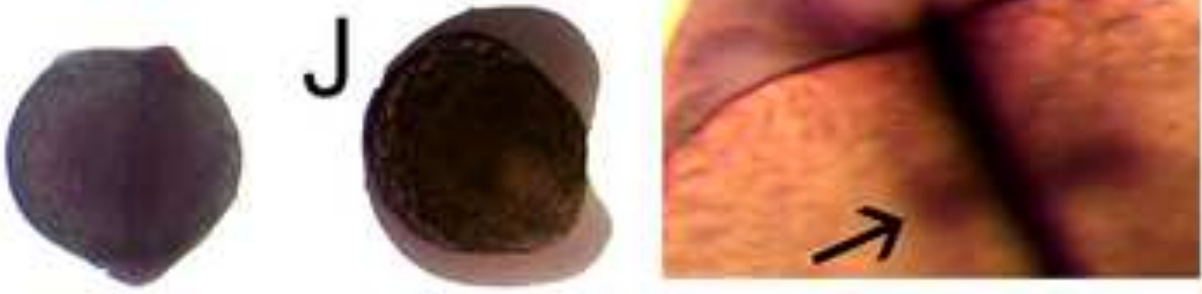


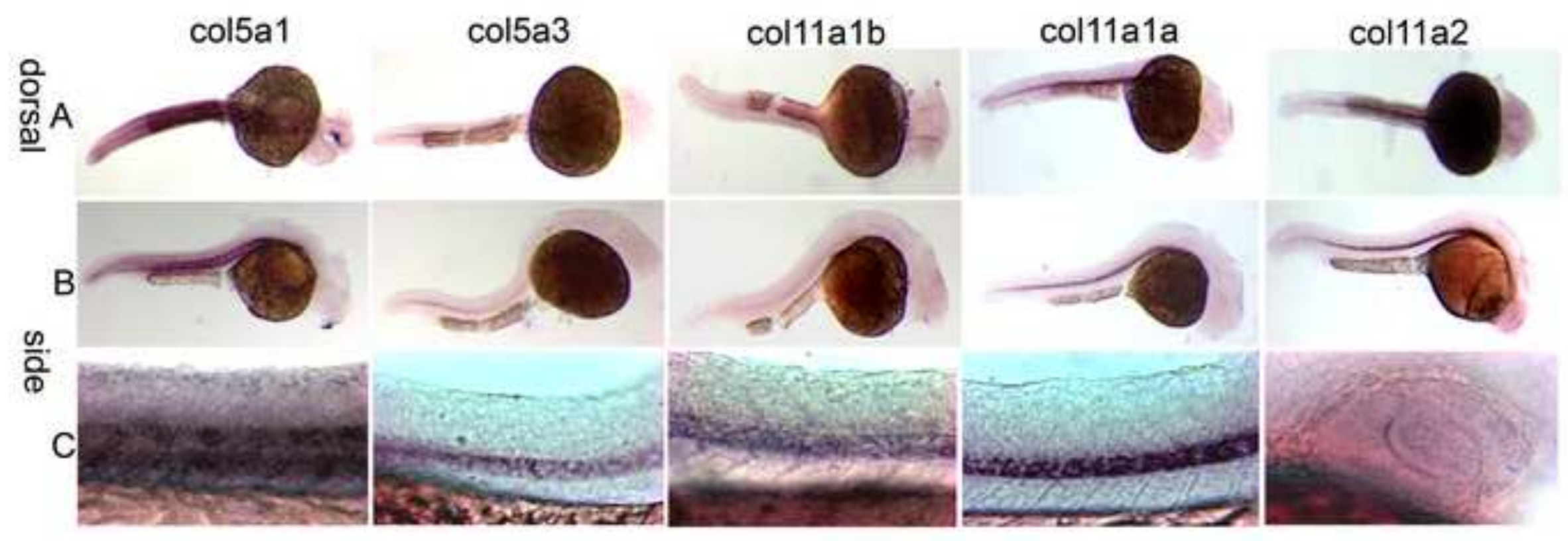




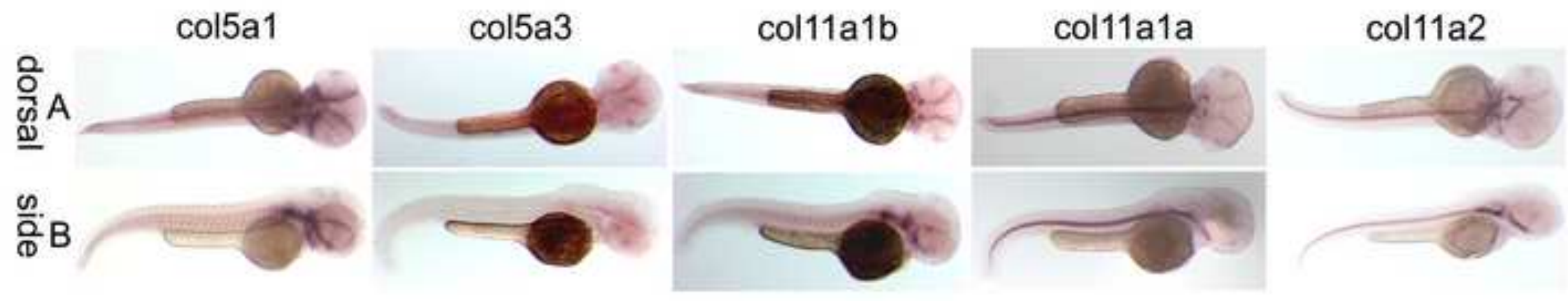



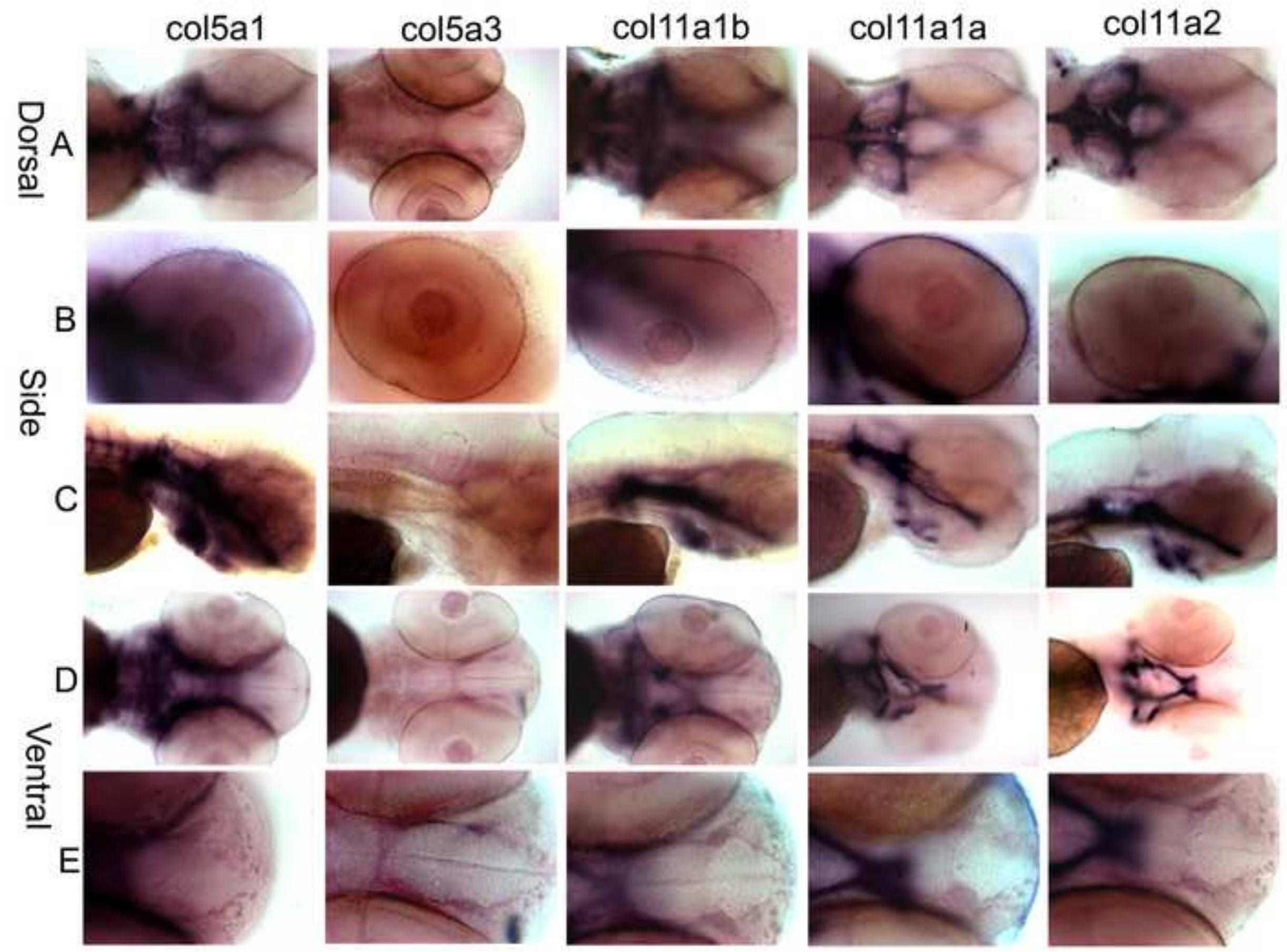